\title{
Correction to: High-resolution melt analysis enables simple genotyping of complicated polymorphisms of codon 18 rendering the NUDT15 diplotype
}

\author{
Yoichi Kakuta $^{1}$ - Yasuhiro Izumiyama ${ }^{1}$ - Daisuke Okamoto ${ }^{1}$. Takeru Nakano ${ }^{1}$. \\ Ryo Ichikawa ${ }^{1}$ - Takeo Naito ${ }^{1}$ Rintaro Moroi $^{1} \cdot$ Masatake Kuroha $^{1}$. \\ Yoshitake Kanazawa $^{1}$ - Tomoya Kimura ${ }^{1}$ Hisashi Shiga ${ }^{1}$ - Hisaaki Kudo ${ }^{2}$. \\ Naoko Minegishi ${ }^{2} \cdot$ Yosuke Kawai $^{3} \cdot$ Katsushi Tokunaga $^{3} \cdot$ Masao Nagasaki ${ }^{4}$. \\ Yoshitaka Kinouchi $^{5} \cdot$ Yasuo Suzuki $^{6} \cdot$ Atsushi Masamune $^{1} \cdot$ for the MENDEL study \\ group
}

Published online: 25 November 2019

(C) Japanese Society of Gastroenterology 2019

\section{Correction to: J Gastroenterol https://doi.org/10.1007/s00535-019- 01638-x}

The correct name of the last author should be "Atsushi Masamune", and not "Atsushi Masasmune" as given in the original publication of the article.

The original article can be found online at https://doi.org/10.1007/ s00535-019-01638-x.

Yoichi Kakuta

ykakuta@med.tohoku.ac.jp

1 Division of Gastroenterology, Tohoku University Graduate School of Medicine, 1-1 Seiryo, Aoba, Sendai 980-8574, Japan

2 Department of Biobank Life Science, Tohoku Medical Megabank Organization, Tohoku University, Sendai, Japan

3 Genome Medical Science Project, National Center for Global Health and Medicine, Tokyo, Japan

4 Human Biosciences Unit for the Top Global Course Center for the Promotion of Interdisciplinary Education and Research (CPIER), Kyoto University, Kyoto, Japan

5 Institute for Excellence in Higher Education, Student Health Care Center, Tohoku University, Sendai, Japan

6 Inflammatory Bowel Disease Center, Toho University Sakura Medical Center, Sakura, Japan 